\title{
ZIVILRECHT UND ZIVILGERICHTSBARKEIT IN DEN FRANKOPHONEN NEUSTAATEN AFRIKAS
}

\author{
Von Christian Kohler
}

Seit Anfang der sechziger Jahre vollzieht sich in den afrikanischen Neustaaten, die aus den ehemaligen französischen Besitzungen und Mandatsgebieten hervorgingen, eine Entwicklung, die zunächst schlagwortartig als Prozeß der Rechtsvereinheitlichung und Rechtserneuerung bezeichnet werden soll1. Ein Jahrzehnt nach Beginn dieser Entwicklung mag ein vergleichender Blick auf den Rechtszustand vor und nach der Unabhängigkeit auch für den europäischen Betrachter lohnen, zumal in der Bundesrepublik erst zögernd begriffen wird, welche Möglichkeiten die den nun souveränen Staaten zugefallene Aufgabe, „ihr“ Recht und „ihre“ Gerichtsbarkeit $\mathrm{zu}$ finden und $\mathrm{zu}$ formen, für eine wirksame Entwicklungshilfepolitik birgt ${ }^{2}$.

Die folgende - freilich nur als Einführung zu lesende - Darstellung ${ }^{3}$ befaßt sich mit den aus den Territorien Französisch-Westafrika und Französisch-Äquatorialafrika hervorgegangenen Staaten, ferner den ehemaligen UN-Treuhandgebieten Togo und Kamerun, soweit sie unter französischer Verwaltung standen, sowie Madagaskar4.

\section{Rechtszustand vor Erlangung der Unabhängigkeit}

Der Unabhängigkeit der ehemaligen afrikanischen Besitzungen ging eine zweijährige Übergangszeit voraus, während der die zukünftigen Staaten durch Gewährung einer weitgehenden inneren Autonomie Gelegenheit haben sollten, einen eigenständigen Staatsapparat $\mathrm{zu}$ organisieren und somit eine antizipierte Staatsgewalt auszuüben. Es war dies die Communauté Institutionelle, die nach der Verfassung der V. Französischen Republik vom 4. Oktober 1958 die Union française von 1946 ablöste. Während dieser Zeit der Autonomie begannen auch die Entwicklungsprozesse, die teilweise zu einer völligen Neuordnung der Gerichtsbarkeit und sogar des materiellen Rechts führten. Diese Neugestaltung des Justizwesens wurde allerdings gemäß Art. 78 der Verfassung von der Communauté überwacht. Eine vergleichende Betrachtung kann sich somit nur auf die Zeit vor dem 4. Oktober 1958 beziehen, als die Organisation der Gerichtsbarkeit wie auch die Setzung des Rechtsanwendungsrechts noch ausschließlich in französischer Hand lagen.

1 Im Rahmen dieses einführenden Uberblicks konnte die vergleichbare Entwicklung in dem ehemals belgischen Congo (Kinshasa) außer Betracht bleiben, vgl. dazu neuerdings Crabb, The Legal System of Congo-Kinshasa, 1970, insbes. $81 \mathrm{ff} ., 105 \mathrm{ff}$.

2 Die 1969 ins Leben gerufene "Forschungs- und Beratungsstelle für die Gesetzgebung der Entwicklungsländer" an der Freien Universität Berlin soll einen Beitrag zu einer so verstandenen Entwicklungshilfe leisten.

3 Zur Vertiefung vgl. in der neueren Literatur insbes. Salacuse, An Introduction to Law in French-Speaking Africa, Vol. I, Africa South of the Sahara, 1969; Lampué, Droit d'outre-mer et de la Coopération, 4. Aufl. 1969, vgl. hierbei jedoch die für den früheren Rechtszustand ergiebigere 3. Aufl. 1959 (zitiert Rolland-Lampué); Luchaire, Droit d'outre-mer et de la Coopération, 2. Aufl. 1966.

4 Die Insel La Réunion ist weiterhin als département d'outre-mer abhängig von Frankreich, vgl. dazu Lampué, a. a. O., S. $144 \mathrm{f}$. 
1. Bis zum Jahre 1958 unterschied man in den hier interessierenden Gebieten zwischen zwei Arten der Gerichtsbarkeit. Es gab zunächst eine justice (juridictions) de droit commun, wobei droit commun das französische Recht war ${ }^{5}$. Auf diesen Begriff ist später, bei der Behandlung des anzuwendenden Rechts, noch einzugehen. Dieser Gerichtsbarkeit - einer Gerichtsbarkeit der Minderheiten - unterfielen zunächst die ortsansässigen Franzosen sowie diejenigen Eingeborenen, die in den Fällen, wo dies möglich war, von dem ihnen zustehenden Wahlrecht bezüglich der Gerichtsbarkeit, unter die sie sich stellen wollten, zugunsten der französischen Gerichtsbarkeit - eben der justice de droit commun - Gebrauch machten ${ }^{6}$.

Diese Wahl bezüglich der Gerichtsbarkeit bedeutete jedoch keine Wahl bezüglich des anzuwendenden Rechts. Hier muß scharf unterschieden werden: eine mögliche Prorogation der Parteien betraf lediglich die Gerichtsbarkeit, somit den formellen Teil des Rechtsstreits. Andererseits war auch unter bestimmten Umständen eine Wahl des anzuwendenden Rechts möglich; eine solche bezog sich stets auf ein oder mehrere Rechtsverhältnisse, somit auf den materiellen Teil des Rechtsstreits. Die Wahl des anzuwendenden Rechts konnte zwar ihrerseits, wenn sie zugunsten des französischen Rechts geschah, die Kompetenz der französischen Gerichtsbarkeit zur Folge haben. Im umgekehrten Fall, wo die Wahl zugunsten des Stammesgewohnheitsrechts ausfiel, hatte dies nicht etwa notwendig die Kompetenz der Eingeborenengerichtsbarkeit zur Folge. Somit waren die Parteien, die sich zwar einer juridiction de droit commun unterworfen hatten, ihr gewohnheitsrechtliches Personalstatut aber beibehielten, nicht etwa nach französischem Recht, sondern vielmehr nach dem auf sie zutreffenden Gewohnheitsrecht zu beurteilen.

Der Aufbau dieser französischen Gerichtsbarkeit glich dem der Gerichtsbarkeit im Mutterland 7 . Es bestanden drei Instanzen. Als erste wurden die tribunaux de première instance tätig, denen allerdings Friedensgerichte - justices de paix vorgeschaltet waren, deren Entscheidungen jedoch vor den ersten Instanzen angefochten werden konnten. Berufungen gegen erstinstanzliche Urteile gingen an das Tribunal supérieur d'appel oder an die Cour d'appel - je nach Territorium. Letzte Instanz war die Cour de Cassation in Paris.

2. Weitaus differenzierter stellt sich nun die Gerichtsbarkeit dar, die nach dem Willen des französischen Gesetzgebers seit dem 3. 12. 1931 als Eingeborenengerichtsbarkeit tätig werden sollte ${ }^{8}$. Hierfür existieren mehrere Bezeichnungen, so z. B. justice (juridictions) de droit local, justice de droit traditionnel, justice de droit coutumier, was hier lediglich mit dem Ausdruck Eingeborenengerichtsbarkeit wiedergegeben werden soll.

Die Organisation dieser Gerichtsbarkeit ist im Laufe der Zeit oftmals umgestaltet worden. So fällt zunächst auf, daß das grundlegende Décret organique vom 3. 12. 1931 kein einziges Gericht vorsah, das ausschließlich mit eingeborenen Richtern besetzt war. Als sich jedoch später herausstellte, daß die erstinstanzlichen Gerichte der Eingeborenengerichtsbarkeit außerordentlich überlastet wurden, entschloß man sich, durch Dekret vom 26. 7. 1944 zusätzlich zu den bereits beste-

\footnotetext{
5 Vgl. hierzu und zum folgenden Pageard, La réforme des juridictions coutumières et musulmanes dans les nouveaux états de l'ouest africain, Penant 1963, S. $462 \mathrm{ff}$.

6 Vgl. Rolland-Lampué, Droit d'outre-mer, 3. Aufl. 1959, S. 216 ff., 218.

7 Vgl. hierzu Pageard, a. a. O., S. $464 \mathrm{f}$

8 Hierzu Rolland-Lampué, a. a. O., S. $383 \mathrm{ff}$.; vgl. auch Wengler, Eingeborenengerichtsbarkeit in den Mandatsgebieten Togo und Kamerun, 1938, Sonderdruck der Zeitschr. f. vergleichende Rechtswissensch., Bd. 58, S. $67 \mathrm{ff}$. (89 ff.).
} 
henden Instanzen der Eingeborenengerichtsbarkeit sog. tribunaux coutumiers zu schaffen, die nun ausschließlich mit eingeborenen Stammeshäuptlingen, Notablen und Beisitzern besetzt wurden. Diese Tribunaux coutumiers erlangten zwar nie sonderliche Bedeutung - ganz im Gegenteil übrigens zu der Situation in den englischen Protektoraten in Afrika - doch wurden sie in Vergleichssachen und auch in der streitigen Gerichtsbarkeit zu einer spürbaren Entlastung der erstinstanzlichen Gerichte der Eingeborenengerichtsbarkeit ${ }^{9}$.

Bemerkenswert ist, daß es sich bei den tribunaux coutumiers um die einzige Art der Rechtsprechung für Eingeborene durch Eingeborene handelt, will man einmal von den in den westafrikanischen Gebieten tätigen Kadi-Gerichten absehen, denen ohnehin eine Sonderstellung zukommt.

Die zweite Kategorie der Rechtsprechung für Eingeborene wird nun von den oben erwähnten juridictions de droit local (coutumier) gebildet. Die Organisation dieser Gerichtsbarkeit war in Französisch-West- und in Französisch-Äquatorialafrika stark unterschiedlich, sowohl in bezug auf die Instanzenzüge, wie auch bezüglich der Mitwirkung eingeborener Beisitzer bei der Urteilsfindung. Der Gerichtsbarkeit im französischen Mutterland - und den Vorstellungen einer ordentlichen Gerichtsbarkeit überhaupt - entsprach am ehesten Französisch-Westafrika, ein Gebiet, das die heutigen Staaten Senegal, Mauretanien, Mali (früher Französisch-Sudan), Niger, Dahomey, Elfenbeinküste, Obervolta und Guinea umfaßte. Hier waren die ersten Instanzen, je nach Streitwert, entweder die tribunaux du premier degré oder die tribunaux du deuxième degré, die auch gleichzeitig Berufungsgerichte waren. Als weiteres Berufungsgericht war das Tribunal supérieur de droit local vorgesehen. Revision war in einigen Fällen möglich zur Chambre d'Annulation.

Den oberen Gerichten saßen weiße Richter vor, den unteren höhere Verwaltungsbeamte; als Beisitzer hatten in jedem Fall zumindest zwei eingeborene Rechtskundige - Notablen - mitzuwirken. Diese hatten beschließende Stimme - voix délibérative.

Dieser verhältnismäßig liberalen Organisation stand diejenige der Eingeborenengerichtsbarkeit in Französisch-Äquatorialafrika gegenüber, die einen ungleich größeren verwaltungsähnlichen Charakter aufwies. Dieses Territorium entspricht den heutigen Staaten Tschad, der Zentralafrikanischen Republik (ehemals OubanguiChari), Gabun und Kongo (Brazzaville). Es gab dort lediglich eine Berufungsinstanz, die tribunaux du deuxième degré; eine dem Tribunal supérieur de droit local ähnliche Rechtsmittelinstanz gab es nicht. Auch hatte die Chambre d'Annulation reinen Verwaltungscharakter, da die Mitwirkung von Richtern, wenn überhaupt welche tätig wurden, auf die tribunaux du deuxième degré beschränkt blieb. So wurde der größte Teil der zur Entscheidung vorliegenden Streitigkeiten rechtskundigen Verwaltungsbeamten überlassen. Allerdings mußten auch bei diesen Gerichten rechtskundige eingeborene Beisitzer, sogenannte assesseurs coutumiers, bei der Urteilsfindung mitwirken. Diese hatten jedoch, im Gegensatz zu Französisch-Westafrika, lediglich beratende Stimme - voix consultative -, so daß es letzten Endes dem Gutdünken des Vorsitzenden anheimgestellt blieb, die von den Eingeborenen vorgebrachten Rechtsansichten anzuerkennen oder nicht.

9 Pageard, 2. 2. O. 
Die Gerichtsbarkeit in den Treuhandgebieten Togo und Kamerun ${ }^{10}$ sowie in Madagaskar entsprach in den wesentlichen Grundzügen derjenigen Französisch-Westafrikas. Bis auf Madagaskar - wo die eingeborenen Beisitzer nur beratende Funktionen ausübten - wurde allgemein der Mitwirkung von Eingeborenen und der Anwendung von Eingeborenenrecht mehr Raum gegeben als etwa in Französisch-Äquatorialafrika.

An dritter Stelle stehen die Kadi-Gerichte (juridictions musulmanes), die mit begrenzter Kompetenz lediglich für Islamiten tätig wurden. Diese Gerichte existierten naturgemäß nur in Französisch-Westafrika, und zwar gemäß Dekret vom 20. 11.1932 für die Bezirke Dakar, Saint-Louis, Gorée und Rufisque. Der Kadi entschied alleine; die Mitwirkung von Kolonialbeamten war nicht vorgesehen ${ }^{11}$.

3. Die Frage, welches Recht anzuwenden war, ist für die Gerichte der juridictions de droit commun verhältnismäßig einfach zu beantworten. Es war dies zunächst das französische Recht, so, wie es für diese überseeischen Gebiete galt. Danach wurden alle Franzosen beurteilt, wie auch diejenigen Eingeborenen, die ganz auf ihr gewohnheitsrechtliches Personalstatut verzichtet hatten und somit ihrem Stammesgewohnheitsrecht nicht mehr unterfielen. Außerdem war französisches Recht dann anzuwenden, wenn von eingeborenen Parteien ein Rechtsgeschäft unter ausdrücklicher Berufung auf das französische Recht vorgenommen wurde; nahm weiterhin ein Eingeborener ein Rechtsgeschäft vor, für das das auf ihn anwendbare Stammesgewohnheitsrecht keine Regelung vorsah, hatte er aber für das französische Recht nicht ausdrücklich optiert, so griff dieses als droit commun dennoch ein. Es füllte somit alle eventuell entstehenden Lücken auch ohne ausdrückliche Zuweisung12. Stammesgewohnheitsrecht wurde vor den französischen Gerichten dann angewendet - wie oben angedeutet wurde - wenn eine option de juridiction zugunsten der französischen Gerichtsbarkeit stattgefunden hatte, die Parteien ihr gewohnheitsrechtliches Personalstatut aber nicht aufgegeben hatten.

Noch einfacher ist die Lage bei den juridictions de droit local: diese wendeten ausschließlich das Gewohnheitsrecht der Parteien an, soweit dieses nicht gegen den vom französischen Recht als droit commun gebildeten ordre public verstieß. Ebenso war die Lage bei den Kadi-Gerichten Französisch-Westafrikas, die nach dem islamischen Recht der malekitischen Schule urteilten.

4. Abschließend sind nun die Konfliktsmöglichkeiten zu erörtern, die sich in der Zeit vor 1958 in zweierlei Hinsicht bemerkbar machen konnten.

Trafen in einem Rechtsstreit ein Eingeborener und ein Franzose bzw. ein einem Franzosen Gleichgestellter zusammen, so war für diesen litige mixte oder Mischprozeß generell das französische Gericht zuständig. Die Parteien konnten allerdings durch schriftliche Erklärung ihren Streit auch vor ein Eingeborenengericht bringen, wobei im ersten Fall die Sache nach französischem, im zweiten Fall nach dem Stammesgewohnheitsrecht des beteiligten Eingeborenen zu entscheiden war.

Eine zweite Konfliktsmöglichkeit konnte sich daraus ergeben, daß die beiden eingeborenen Parteien, die ihren Streit entweder dem französischen oder dem zuständigen Eingeborenengericht vorgelegt hatten, nach verschiedenen Gewohnheitsrechten zu beurteilen waren. Man sprach hierbei von einem sogenannten conflit de

10 Hierzu eingehend Wengler, a. a. O., S. $89 \mathrm{ff}$.

$11 \mathrm{Vgl}$. Mangin, L'Organisation judiciaire des Etats d'Afrique et de Madagascar, Revue juridique et politique d'outre-mer 1962 , S. $80 \mathrm{ff}$. (87).

$12 \mathrm{Vgl}$. hierzu und zum folgenden Luchaire, Droit d'outre-mer, 1959, S. $343 \mathrm{f}$. 
coutumes, den man nach Grundsätzen eines intergentilen Privatrechts zu lösen suchte. Einige dieser Kollisionsregeln waren durch Dekrete vom 21. 4. 1933 und 3. 12. 1931 für einige Gebiete Französisch-Westafrikas aufgestellt worden. So fand in Ehesachen dasjenige Recht Anwendung, nach dem der Ehevertrag geschlossen wurde oder, wenn dies nicht der Fall war, das Recht der Frau. In Erbsachen und bei Schenkungen war das Recht des Erblassers bzw. des Schenkers, bei Verträgen die lex loci contractus maßgebend. In Zweifelsfällen wurde das Recht des Beklagten angewendet ${ }^{13}$.

\section{Gerichtsbarkeit und anwendbares Recht in den Neustaaten}

1. Bei der Untersuchung des Rechtszustandes in den Neustaaten Schwarzafrikas nach Erlangung der Unabhängigkeit ist im folgenden - soweit die Neuordnung der Gerichtsverfassung und die Schaffung „eigenen“, autochthonen materiellen Rechts betroffen sind - insbesondere zu fragen, wieweit die vom französischen Gesetzgeber entwickelten Prinzipien unmittelbar weiterwirken oder in veränderter Form erneut bemerkbar werden, bzw. inwieweit von den Neustaaten eigenständige Vorstellungen entwickelt und durchgesetzt werden. Auf dem Gebiet der Gerichtsverfassung boten sich den Gesetzgebern der neugebildeten Staaten zwei Möglichkeiten: es konnte die bisherige Zweispurigkeit der Gerichtsbarkeit beibehalten oder versucht werden, eine einheitliche Gerichtsbarkeit zu schaffen, die dann jedem, ohne Rücksicht auf das im Einzelfall anwendbare Recht, offenstand.

Die naheliegenden Bemühungen, sich des kolonialen „Erbes“ auf diesem Gebiet zu entledigen - dies teilweise mit Hilfe wenig durchdachter Scheinlösungen ${ }^{14}$ haben dazu geführt, daß im Jahre 1970 von den insgesamt 15 Neustaaten lediglich drei den dualistischen Aufbau der Gerichtsbarkeit beibehalten haben, nämlich aus dem ehemaligen Territorium Französisch-Westafrika Mauretanien und Obervolta, außerdem das ehemalige Treuhandgebiet Togo. Tendenzen, später eine einheitliche Gerichtsbarkeit zu organisieren, bestehen im übrigen auch in Obervolta und in Togo, so daß die derzeitige Gerichtsverfassung dieser Staaten wohl nur als Übergangslösung anzusehen sein wird ${ }^{15}$.

a) Die Tatsache, daß Mauretanien Bestrebungen zur Vereinheitlichung der Gerichtsbarkeit nicht kennt, darf nun nicht zu dem Schluß verleiten, dort werde das seinerzeit von der Kolonialmacht eingerichtete System als den Bedürfnissen des Landes angemessen empfunden. Es ist vielmehr so, daß das ehemalige System der Kolonialzeit nur die äußere Form hergab, in die das neue Gerichtsverfassungsrecht gegossen wurde.

Durch Gesetz vom 27. 6. $1961^{16}$ wurden zwei Gerichtsbarkeiten ins Leben gerufen, und zwar eine justice de droit musulman sowie eine justice de droit moderne. Die scharfe Trennung dieser beiden Gerichtsbarkeiten entspricht der Struktur

13 Vgl. Pageard, Penant 1963, S. $479 \mathrm{f}$.

14 Hier sei nur an die radikale "Rechtsvereinheitlichung“ in der Republik Guinea (siehe dazu unten S. $129 \mathrm{f}$.) und an die Nebenwirkungen der Vereinheitlichung der Gerichtsbarkeit in Kamerun (dazu unten S. 135) erinnert.

15 Vgl. Pageard, Penant 1963, S. 483, der zum Teil - insbesondere bezüglich des Tschad und Dahomeys durch die seit 1963 eingetretene Entwicklung überholt ist; in Ober-Volta ist allerdings durch Gesetz $\mathrm{Nr}$. 9-63 vom 10.5. 1963 das Bestehenbleiben der Juridictions de Droit Coutumier ausdrücklich bestätigt worden, vgl. noch Marchand, L'Organisation judiciaire en Haute-Volta, Penant 1964, S. $121 \mathrm{ff}$.

16 No. 61-123, geändert durch Gesetz No. 62-153 vom 10. 7. 1962; vgl. hierzu eingehend Jeol, Cours de droit judiciaire mauritanien, 1965. 
Mauretaniens, das sich selbst als République Islamique de Mauritanie bezeichnet und wo der Islam Staatsreligion ist. Die Gerichtsbarkeit des islamischen Rechts umfaßt die Kadi-Gerichte, die den sogenannten juridictions de droit musulman de première instance vorgeschaltet sind. Sie werden hauptsächlich in Vergleichssachen tätig und haben in den übrigen Fällen Kompetenz nur bis zu einem bestimmten Streitwert. Auf die juridictions de première instance folgt nunmehr, nach einer Reorganisation der Gerichtsverfassung im Jahre $1965^{17}$ als einzige Rechtsmittelinstanz die Cour Suprême, die auch für Rechtsmittel gegen Entscheidungen der KadiGerichte zuständig ist.

Die Gerichtsbarkeit des modernen Rechts umfaßt dagegen lediglich die juridiction de droit moderne de première instance und die Cour Suprême. In der Gerichtsverfassung Mauretaniens nimmt allerdings diese justice de droit moderne eine Ausnahmestellung ein. Das ergibt sich aus den die Kompetenzzuweisung regelnden Bestimmungen des Code de procédure civile, commerciale et administrative vom 2. 2. 1962. Dort bestimmt Art. 1: „Les juridictions de droit musulman connaissent de toutes les affaires civiles et commerciales sous réserve des dispositions de l'article suivant."

Dieser generell die sachliche Zuständigkeit der Gerichte islamischen Rechts regelnden Vorschrift folgt im nächsten Artikel eine limitative Aufzählung der Fälle, in denen die Gerichte modernen Rechts zuständig sind. Es handelt sich hierbei im wesentlichen um Enteignungssachen, Fragen des Staatsangehörigkeitsrechts, Handelssachen, wenn eine der beteiligten Gesellschaften nach modernem Recht errichtet wurde. Dazu kommen noch die Fälle, in denen die Parteien zulässigerweise modernes Recht für anwendbar erklärt haben.

Aus diesen beiden Vorschriften ergibt sich zwingend - da ausdrückliche anderweitige Regelungen nicht vorhanden sind - die Zuständigkeit der juridictions de droit musulman auch bei sogenannten litiges mixtes, in denen eine Partei unter normalen Umständen der Gerichtsbarkeit modernen Rechts unterworfen wäre. Eine option de juridiction zugunsten der juridictions de droit moderne ist nicht zulässig. Ein solches Wahlrecht würde zudem einen Bruch in der betont nationalen und etwaigen nachwirkenden Einflüssen des Rechtszustandes vor der Unabhängigkeit ablehnend gegenüberstehenden Gerichtsverfassung Mauretaniens bedeuten ${ }^{13}$.

Aus dem bisher Gesagten ergibt sich auch die Feststellung des anwendbaren Rechts. Die Rolle des droit commun wird vom islamischen Recht der malekitischen Schule übernommen. Das in den wenigen oben aufgezählten Fällen anwendbare moderne Recht ist das französische, so wie es für Mauretanien - das heißt für Französisch-Westafrika - bis zum Jahre 1960 galt. Das Verhältnis der beiden Rechtsordnungen zueinander ist somit genau umgekehrt wie vor der Entkolonialisierung. Es entspricht der neuen Struktur der Gerichtsverfassung des Landes.

Eventuelle Konflikte bezüglich des anzuwendenden Rechts werden zugunsten des droit commun - also des islamischen Rechts - gelöst. Eine option de législation ist außer in einigen wenigen Fällen ebensowenig wie eine option de juridiction möglich. Es zeigt sich somit bei Beibehaltung der Zweispurigkeit sowohl der Gerichtsbarkeit wie auch der Statuten eine deutliche Bevorzugung der autochthonen

17 Gesetz No. 65-123 vom 20.7. 1965; dieses Gesetz gibe im wesentlichen die gerichtsverfassungsrechtlichen Bestimmungen der Gesetze von 1961 und 1962 wieder, schafft aber - hierin liegt seine eigentliche Bedeutung - die Tribunaux supérieurs d'appel ab.

$18 \mathrm{Vgl}$. etwa die Außerungen von Jeol, a. a. O., S. 34, und in La réforme de la Justice en République Islamique de Mauritanie, Penant 1962, S. $193 \mathrm{ff}$. 
Rechtsordnung. Im übrigen ist eine Kodifikation des Zivilrechts in Vorbereitung; man beabsichtigt, die zur Zeit noch abwendbaren Bestimmungen des französischen Rechts in die nationale Rechtsordnung $\mathrm{zu}$ integrieren ${ }^{\mathbf{1 0}}$.

b) Die Gerichtsverfassung der beiden übrigen Staaten, die das „système dualiste“ beibehalten haben, entspricht hingegen im wesentlichen dem Aufbau der Gerichtsbarkeit vor der Unabhängigkeit. In der Republik Ober-Volta ${ }^{20}$ beginnt der Instanzenzug der Gerichtsbarkeit des „modernen“, d. h. immer noch des französischen Rechts, bei den Tribunaux de 1 ère Instance, von wo aus die Berufung zur Cour d'Appel gegeben ist; pourvoi en cassation bzw. pourvoi en revision ist schließlich zur Chambre judiciaire der Cour Suprême möglich. Für die Eingeborenengerichtsbarkeit - juridictions de droit local - sind Tribunaux du $1^{\text {er }}$ degré und Tribunaux du $2^{\text {ème }}$ degré eingerichtet. Urteile der letztgenannten Gerichte sind mit der Berufung bei der Cour d'Appel anfechtbar; im übrigen kennt auch die Eingeborenengerichtsbarkeit den pourvoi en cassation zur Cour Suprême.

Der Stand des materiellen Rechts in Ober-Volta - Zweiteilung der Statuten mit den oben erwähnten Konfliktlösungen -- ist seit der Unabhängigkeit im wesentlichen unverändert.

Die Gerichtsverfassung in der Republik Togo'1 weist gegenüber Ober-Volta kaum nennenswerte Abweichungen auf. Einer Gerichtsbarkeit modernen Rechts mit drei Instanzen steht eine Eingeborenengerichtsbarkeit gegenüber, deren Instanzenzug die Tribunaux coutumiers de 1 ère Instance und die Tribunaux coutumiers d'Appel umfaßt; von diesen Gerichten ist der pourvoi en annulation zur Cour d'Appel des modernen Rechts gegeben. Erwähnenswert ist, daß die Tribunaux coutumiers mit gewohnheitsrechtskundigen Beisitzern besetzt sind, die beschließende Stimme haben. Auch in Togo ist trotz neuerer Bestrebungen zur Rechtsvereinheitlichung der Stand des materiellen seit der Unabhängigkeit kaum verändert ${ }^{22}$.

2. Die übrigen zwölf Staaten beschritten - zumindest bezüglich der Gerichtsverfassung - einen neuen Weg, indem sie sich für den einheitlichen Aufbau der Gerichtsbarkeit, für das „système unitaire“, entschieden. Einige dieser Staaten reorganisierten ihre Gerichtsverfassung alsbald nach der Unabhängigkeit im Sinne dieses Modells, andere - von denen noch zu sprechen sein wird - behielten zunächst über längere Zeit den zweispurigen Aufbau der Gerichtsbarkeit bei.

a) Eine Sonderstellung unter den Staaten des „système unitaire“ nimmt die sozialistische Republik Guinea ein. Das unorthodoxe und tatsächlich revolutionäre Vorgehen des guineischen Gesetzgebers sowie die Unvergleichbarkeit mit den übrigen Staaten verdienen Hervorhebung. Guinea, das schon 1958 nach Inkrafttreten der Verfassung der V. Republik erklärte, der Communauté Institutionelle nicht beitreten $\mathrm{zu}$ wollen, sondern als einziges Land die sofortige Unabhängigkeit wählte, nahm durch zwei Gesetze - vom 29. 12. 1960 und 14. 4. 1962 - eine völlige Umgestaltung der Gerichtsverfassung vor ${ }^{23}$. Die beiden Gerichtsbarkeiten,

19 Jeol, Cours de droit judiciare mauritanien, S. 35.

20 Loi No. 9-63 vom 10.5. 1963, vgl. auch Marchand, L'Organisation judiciaire en Haute-Volta, Penant 1964 , S. $121 \mathrm{ff}$

21 Loi No. 61-17 vom 12. 6. 1961.

22 Vgl. noch Amega, Prière pour un Code civil, Penant 1966, S. $275 \mathrm{ff}$; A Acouetey, Unité ou dualité des statuts dans le droit de la famille au Togo, Revue juridique et politique 1967, S. $34 \mathrm{ff}$.

23 Vgl. hierzu und zum folgenden Voss, Guinea, 1968, S. 109 ff., und den folgenden Artikel desselben Verfassers in diesem Heft. 
die bis 1958 Recht sprachen, traten danach außer Funktion; an ihre Stelle trat eine einheitliche Gerichtsbarkeit, die der Verwirklichung der Losung der guineischen Einheitspartei „Un seul Peuple, une seule Justice“ dienen sollte. Die Tribunaux de droit indigèn $e^{24}$ wurden durch die Tribunaux populaires du $1^{\text {er }}$ und du $2^{\text {me }}$ degré ersetzt, deren Zuständigkeit jedoch alsbald auf Personenstandssachen und auf vorprozessuale Schlichtung in Zivil- und Handelssachen eingeschränkt wurde ${ }^{25}$. Weiter wurde in jeder der 29 Regionen eine Justice de Paix à compétence étendue eingerichtet, ferner 4 Tribunaux de 1 ère Instance; als Berufungsinstanz für sämtliche Entscheidungen der eben genannten Gerichte ist die Cour d'Appel de la République de Guinée in Conakry vorgesehen. Eine richterliche Unabhängigkeit im herkömmlichen Sinne gibt es nicht; die Richter als „travailleurs de la Justice“ haben „militants comme les autres“ $\mathrm{zu}$ sein und dem sozialistischen Aufbau zu dienen ${ }^{26}$.

b) Von den übrigen - 1960 unabhängig gewordenen - Staaten entschlossen sich während der ersten drei Jahre nach Erlangung der Unabhängigkeit bereits acht für eine Reorganisation der Gerichtsverfassung im Sinne des „système unitaire": Es waren dies aus dem Gebiet des ehemaligen Französisch-Westafrika die Staaten Senegal, Mali, Niger und die Elfenbeinküste; aus dem ehemaligen Territorium Französisch-Äquatorialafrika die Staaten Gabun und Kongo (Brazzaville) sowie das ehemalige Treuhandgebiet Kamerun, sowie Madagaskar.

In den neuen Gerichtsverfassungen dieser Staaten lassen sich wesentliche übereinstimmende Merkmale erkennen. Die neuen Gerichtsbarkeiten stellen in der Regel eine Fusion der ehemaligen juridictions de droit commun und der juridictions de droit local dar. Dabei wird der äußere Aufbau der Kolonialgerichtsbarkeit beibehalten. Wesentlichen Elementen der früheren Eingeborenengerichtsbarkeit wird dadurch Rechnung getragen, daß man Friedens- oder Vergleichsgerichte dem normalen Instanzenzug vorschaltet. Darüber hinaus wird den Vertretern der einzelnen Stammesgewohnheitsrechte, soweit diese zur Anwendung gelangen, ein Mitwirkungsrecht bei der Urteilsfindung gegeben, dem allerdings, wie noch $\mathrm{zu}$ untersuchen sein wird, nicht immer nennenswerte Bedeutung zukommt ${ }^{27}$.

aa) Am Beispiel Senegals soll im folgenden der typische Aufbau einer dem système unitaire zuzurechnenden Gerichtsverfassung gezeigt werden. Rechtsgrundlage ist die Ordonnance No. 60-56 vom 14. 11. 1960, bestätigt durch Gesetz No. 61-53 vom 23. 6. 1961. Danach sind Gerichte des ersten Rechtszuges die Tribunaux de première instance, Berufungsgericht die Cour d'appel, Kassationsgericht die Cour Suprême. Außer diesen drei Instanzen der ordentlichen Gerichtsbarkeit werden Friedensgerichte - justices de paix - tätig, denen in der Praxis außerordentliche Bedeutung zukommt. Ihre sachliche Zuständigkeit liegt im wesentlichen in der Behandlung von Ehe- und Kindschaftssachen, Erbfolge- und Schenkungssachen sowie Grundstückssachen, soweit es sich nicht um eingetragene Grundstücke handelt. In sonstigen Angelegenheiten hängt ihre Zuständigkeit vom Streitwert ab.

\footnotetext{
24 Nach dem Bulletin du Ministère de la Justice Nr. 1/1965, S. 14, ninstrument d'injustice, de vol et surtout de division".

25 Runderlasse Nr. 016 - P. R. G. vom 2.7.1963; Nr. 009 - P. R. G. vom 11. 2. 1964

26 Vgl. das bei Voss, a. a. O., S. 111, zitierte Rundschreiben des Präsidenten der Republik vom 2.7 .1963$. 27 Vgl. Mangin, L'Organisation judiciaire . . , S. 85; Pageard, Penant 1963, S. 492.
} 
Art. 1 der oben zitierten ordonnance bestimmt ausdrücklich, wer dieser Gerichtsbarkeit unterfällt: „... ces juridictions connaissent, quel que soit le statut du justicable, de toutes affaires civiles, commerciales ou pénales et de l'ensemble du contentieux administratif ${ }^{23}$."

Eine einheitliche Gerichtsbarkeit ist somit zuständig sowohl für die Rechtsunterworfenen des statut moderne wie auch des statut traditionnel, das heißt für diejenigen Personen, die einem der siebzig verschiedenen Stammesgewohnheitsrechte unterfallen. Die einzige Ausnahme in dieser Beziehung bilden die Personen mit islamischem Personalstatut. Für sie ist - allerdings nur auf der Basis der Friedensgerichte - ein Kadi zuständig. Diese islamische Gerichtsbarkeit - die einzige in Senegal - bildet einen Teil der Friedensgerichte, ist somit auch nur in den Angelegenheiten zuständig, in denen die Friedensgerichte generell kraft ausdrücklicher Zuweisung Kompetenz haben ${ }^{29}$. Das Prinzip der einheitlichen Gerichtsbarkeit bleibt also auch auf dieser Ebene gewahrt.

In den Friedensgerichten wird Recht durch einen Einzelrichter gesprochen, ebenso bei den tribunaux de première instance. Die Cour d'appel und die Cour Suprême entscheiden in Kollegialbesetzung. Darüber hinaus ist gem. Art. 5 der ordonnance vom 14.11.1960 die Hinzuziehung von zwei gewohnheitsrechtskundigen Beisitzern - assesseurs coutumiers - in sämtlichen Instanzen zwingend vorgeschrieben, wenn die Parteien ihr statut traditionnel beibehalten haben, wie das z. B. für das Recht der Ehe, des Personenstandes, der Erbfolge und Schenkung sowie das Immobiliarrecht möglich ist. Diese Beisitzer haben jedoch lediglich beratende Stimme - voix consultative. In den Friedensgerichten macht naturgemäß der Kadi eine Ausnahme - es wird regelmäßig nach dem islamischen Recht der malekitischen Schule geurteilt - der nur mit einem Beisitzer der ebenfalls Kadi sein muß, Recht spricht.

bb) Bis auf die Besonderheit der Kadi-Rechtsprechung entsprechen die Gerichtsverfassungen der übrigen Staaten, die sich für das système unitaire entschieden haben, derjenigen Senegals, abgesehen von meist unwesentlichen Abweichungen in der Nomenklatur. So entsprechen z. B. im Congo den Friedensgerichten die tribunaux d'instance, in Kamerun die tribunaux de conciliation. Die tribunaux de grande instance erfüllen die gleiche Funktion wie die tribunaux de première instance in den übrigen Staaten.

Eine einheitlich strukturierte Gerichtsorganisation verbietet naturgemäß das Vorhandensein von Gerichtsbarkeiten des Stammesgewohnheitsrechts. Nichtsdestoweniger haben einige Staaten tribunaux coutumiers beibehalten, so Congo durch Gesetz vom 11.1.1961, Kamerun durch Dekret vom 18.12.1959. Zwar sollte dies ausdrücklich als Übergangsregelung gelten; diese Gerichtsbarkeiten bestehen jedoch noch heute ${ }^{30}$.

Diese Besetzung der Gerichte entspricht im übrigen dem senegalesischen Modell. Hier macht nur Kamerun eine Ausnahme, wo bereits die tribunaux de première instance in Kollegialbesetzung entscheiden.

28 Vgl. hierzu Forster, La place de la coutume dans le droit moderne de la Republique du Sénégal, Zeitschr. f. vergl. Rechtswiss. 1964, S. 1 ff.

29 Mangin, a. a. O., vgl. auch das Dekret vom 20.11. 1932

30 Vgl. zur Lage in Kamerun Salacuse, An Introduction. . . , s. $291 \mathrm{f}$. 
Lediglich die Rolle der gewohnheitsrechtskundigen Beisitzer hat in den einzelnen Staaten mit einheitlicher Gerichtsbarkeit eine unterschiedliche Behandlung erfahren. Wie bereits erwähnt, fällt ihnen nach der senegalesischen Gerichtsverfassung eine nur beratende Tätigkeit zu. Das gilt auch für Niger, Kamerun und Gabun. Dagegen nehmen die Beisitzer in Mali, Elfenbeinküste und Congo eine den Richtern gleichgeordnete Stellung ein; sie haben kraft Gesetzes beschließende Stimme.

c) Drei weitere Staaten haben sich in den Jahren bis 1967 für das „système unitaire" entschieden. In der Republik Dahomey wurden durch Gesetz vom 9.12. 1964 die tribunaux coutumiers abgeschafft, ihre Zuständigkeiten gingen zusammen mit denen der bisherigen juridictions de droit moderne - auf tribunaux de première instance über; entsprechend wurden die Rechtsmittelinstanzen vereinheitlicht ${ }^{31}$. In der Zentralafrikanischen Republik, in der in den Jahren 1961 und 1962 eine zweispurige Gerichtsbarkeit eingerichtet worden war ${ }^{32}$, wurden im Zuge der Reorganisation der Gerichtsverfassung im Jahre 1965 und $1966^{33}$ die juridictions de droit traditionnel - allerdings unter Beibehaltung des stammesgewohnheitsrechtlichen Schlichtungsverfahrens - aufgelöst. Die Aufgaben der beiden früheren Gerichtsbarkeiten werden nunmehr von einer einzigen Gerichtsbarkeit nach dem Modell der übrigen Staaten "wahrgenommen. In der Republik Tschad war nach der Erlangung der Unabhängigkeit überhaupt nichts geändert worden; sowohl die dualistische Gerichtsverfassung als auch die Grundsätze des kolonialen Rechtsanwendungsrechts aus der Zeit vor 1958 galten unverändert fort. Ein knappes Jahrzehnt später wurde jedoch durch ordonnance vom 21. 3.1967 eine dem „système unitaire“ zugehörige Gerichtsverfassung mit einer einheitlichen Gerichtsbarkeit eingerichtet, der nunmehr alle Rechtssuchenden unterfallen. Soweit allerdings nach den herkömmlichen Rechtsanwendungsregeln - von einer Rechtsvereinheitlichung kann auch im Tschad noch nicht gesprochen werden Eingeborenenrecht anwendbar ist, werden die erkennenden Gerichte durch gewohnheitsrechtskundige Beisitzer ergänzt ${ }^{34}$.

d) Die Zweiteilung der Statuten ist auch in den Ländern mit einheitlicher Gerichtsbarkeit - mit der oben besprochenen Ausnahme Guineas - nicht beseitigt worden. Vielmehr bestehen eine Vielzahl von Stammesgewohnheitsrechten sowie gesetztes Recht nebeneinander; in einigen Staaten findet darüber hinaus noch Islamrecht Anwendung. Wenn auch nach dem programmatisch erklärten Willen der Gesetzgeber der Neustaaaten die Rechtsvereinheitlichung der Vereinheitlichung der Gerichtsbarkeit folgen soll, so ist doch in der Regel mit der Verwirklichung derartiger Bestrebungen in absehbarer Zeit nicht zu rechnen. Als Ausnahmen von dieser Regel sind jedoch die Beispiele Senegals und Madagaskars hervorzuheben, die als erste der hier interessierenden Staaten auf dem Gebiet des Privatrechts rechtsvereinheitlichend tätig geworden sind. In Senegal konnte nach umfangreichen Vorarbeiten $^{35}$ am 10.7.1963 der Erste Teil (Partie générale) eines einheitlichen

\footnotetext{
31 Vgl. hierzu Adandé, L'Organisation judiciaire et l'évolution législative générale au Dahomey, Penant 1965 , S. $429 \mathrm{ff}$

32 Gesetze No. 61-182, 61-183 vom 17./23. 1. 1961; No. 62-308 vom 21. 5. 1962.

33 Gesetz No. 65-75 vom 23. 12. 1965 und Ord. No. 66-60 vom 30. 8. 1966.

$34 \mathrm{Im}$ übrigen besteht eine Kassationsinstanz nicht mehr, als Rechtsmittelinstanz ist vielmehr lediglich die Cour d'Appel in Fort-Lamy vorgesehen, vgl. R. B., L'Organisation judiciaire au Tchad, Penant 1968, S. $65 \mathrm{ff}$. 35 Vgl. dazu Decottignies, Réflexions sur le projet du Code sénégalais des obligations, Penant 1962, S. 495 ff.;
Forster, La place de la coutume . . . S. 7.
} 
Code sénégalais des obligations verabschiedet werden ${ }^{36}$, der dann zusammen mit dem am 13.7.1966 verabschiedeten $Z$ weiten Teil (Contrats spéciaux) ${ }^{37}$ am 1. 1.1967 in Kraft trat. Die Arbeiten zu den drei weiteren Teilen (Sociétés commerciales; Effets du commerce; Guaranties et Sûretés du créancier) sind noch nicht abgeschlossen. Die verabschiedeten Teile des Code finden nunmehr - unabhängig vom statut personnel des Rechtsuchenden - auf alle ihm unterfallenden Rechtsstreitigkeiten Anwendung; eine Berücksichtigung der bisher grundsätzlich anwendbaren Gewohnheitsrechte ist insoweit ausgeschlossen $^{38}$. Zu der Frage, ob das Ziel des Gesetzgebers, modernes Recht und Gewohnheitsrecht harmonisierend $\mathrm{zu}$ vereinheitlichen, tatsächlich erreicht ist, kann freilich abschließend noch nicht Stellung genommen werden; Salacuse ${ }^{39}$ jedenfalls bezeichnet den Einfluß des Gewohnheitsrechts auf den - letztendlich doch von den Technikern des modernen Rechts konzipierten - Code als „negligible“.

In Madagaskar begannen die schon 1957 beschlossenen Vorarbeiten für einen einheitlichen Code civil malgache im Jahre 1960 mit ausführlichen Bestandsaufnahmen der auf eine lange Tradition gestützten madegassischen Gewohnheitsrechte, die, in einem Rapport de synthèse zusammengefaßt, zur Grundlage der Beratungen wurden ${ }^{40}$. Inzwischen sind bereits - wenn auch der Abschluß des Gesamtwerkes noch nicht vorausgesehen werden kann - mit den gleichen Rechtsfolgen der Vereinheitlichung wie in Senegal wesentliche Teile des vereinheitlichten Zivilrechts in Kraft getreten, so neben einem Allgemeinen Teil das Personenstands- und Familienrecht sowie ein allgemeiner Teil des Obligationenrechts, in dem sich neben der Behandlung der allgemeinen Lehren eine Regelung der Geschäftsführung ohne Auftrag und der ungerechtfertigten Bereicherung finden.

In den übrigen Staaten, aber auch in Senegal und Madagaskar auf dem weiten Gebiet des noch nicht vereinheitlichten Rechțs, ergibt sich aus der immer noch vorhandenen Rechtsvielheit die Notwendigkeit, Regeln über das im Einzelfall anzuwendende Recht - sei es durch Gesetz oder durch die Rechtsprechung - aufzustellen.

Bis zum Jahre 1960 galt das französische Recht als droit commun. Es übte in seinem Geltungsbereich einen Einfluß aus, den René David mit dem des römischen Rechts in Europa bis zum Anbruch des 19. Jahrhunderts verglich ${ }^{41}$. Mit der Entkolonialisierung verlor das französische Recht zwar begrifflich diese Stellung; es fiel jedoch nicht etwa ersatzlos fort, sondern fand auf verschiedenen Wegen Eingang in die neu entstehenden Rechtsordnungen der einzelnen unabhängigen Staaten.

So heißt es etwa in der - hier für die Verfassungen fast aller Neustaaten stehenden - senegalesischen Verfassung ausdrücklich: „Les lois et règlements actuellement en vigueur lorsqu'ils ne sont pas contraires à la présente Constitution, resteront en vigueur tant qu'ils n'auront pas été modifiés ou abrogés.“

Für Gabun wurde dies unter anderem expressis verbis von der Rechtsprechung bebekräftigt. In einer Entscheidung vom 8.1.1963 stellt die Cour d'appel fest: „Le droit français est devenu dès l'avènement de l'indépendance du droit

36 Gesetz No. 63-62.

37 Gesetz No. 66-70.

38 leg. cit., Dispositions finales.

39 op. cit., S. 232.

40 Vgl. hierzu und zum folgenden Salacuse, op. cit. S. $412 \mathrm{ff}$. mit Nachw.; Ramangasoavina, Du droit coutumier aux codes modernes malgaches, Penant 1962, S. $193 \mathrm{ff}$.

41 David, La refonte du Code civil dans les Etats africaines, Penant 1962, S. $352 \mathrm{ff}$. 
gabonais"12. Damit wurde das französische Recht in den neuen Staaten rezipiert. Eine solche Rezeption geschah teils ausdrücklich, teils kann man — wie im Falle Guineas - eine konkludent zugelassene Weitergeltung des französischen Rechts annehmen. Das wird im Falle Guineas dadurch erleichtert, als dort - wie oben erwähnt - das gesamte Stammesgewohnheitsrecht für in der Folge unanwendbar erklärt wurde, eine eigenständige, die entstehenden Lücken füllende Kodifikation aber nicht vorhanden war.

Eine derart vollzogene Rezeption bedeutet nun nicht ohne weiteres, daß das rezipierte französische Recht weiterhin in den einzelnen Staaten droit commun geblieben ist. Für die Länder mit zweispuriger Gerichtsbarkeit wurde dies am Beispiel Mauretaniens, wo der Platz des droit commun nunmehr vom islamischen Recht eingenommen wird, französisches Recht aber nur in Ausnahmefällen gilt, deutlich. Parallele Entwicklungen sind allerdings in den Staaten mit einheitlicher Gerichtsbarkeit nicht festzustellen. So ist z. B. für Gabun ausdrücklich ausgesprochen worden, daß das französische, durch Rezeption gabonesische Recht das droit commun sei ${ }^{43}$. Für die übrigen Staaten gilt dies in dieser Ausschließlichkeit nicht, jedenfalls sind die Formulierungen dort vorsichtiger. Allgemein wird gesagt, daß das "gesetzte Recht“ das droit commun darstelle. „La loi constitue le droit commun."44 Auf dem Gebiet des Zivilrechts bestätigt dies allerdings noch immer die Stellung des französischen Rechts als droit commun. Dem steht nicht entgegen, daß eine derartige Geltung des Code civil ihr Ende bei Inkrafttreten einer einheitlichen eigenständigen Kodifikation fände, wie dies in Senegal und Madagaskar teilweise bereits eingetreten ist ${ }^{45}$. Man wird die Stellung des rezipierten französischen Rechts im Verhältnis zum gesetzten originären Recht des rezipierenden Staates immer als subsidiär ansehen müssen.

Aus dem Charakter des droit commun ergibt sich, daß es grundsätzlich auf jeden Anwendung findet, der der staatlichen Gerichtsbarkeit unterworfen ist. Jedoch haben alle Staaten - Guinea bildet wieder eine Ausnahme - die Anwendbarkeit von Stammesgewohnheitsrecht in verschiedenem Umfang zugelassen. Gleichlautende positive Regelungen zu dieser Frage finden sich für Senegal in Art. 10 der ord. vom 14.11. 1960 sowie für Niger in Art. 51 des Gesetzes vom 16.3.1962. Diese Bestimmungen zählen limitativ die Fälle auf, in denen Stammesgewohnheitsrecht anwendbar ist. Der Katalog umfaßt das Recht der Geschäftsfähigkeit, des Personenstands, der Ehe und Scheidung, der Kindschaft, Erbfolge und Schenkung. Auch zählt das Immobiliarrecht hierzu, soweit es sich nicht um eingetragene Grundstücke handelt, oder um solche, deren Erwerb und Verlust durch Gesetz geregelt ist (Art. 12 bzw. Art. 51). Selbst das Vorliegen dieser Voraussetzungen begründet noch nicht ohne weiteres die Anwendbarkeit des Stammesgewohnheitsrechts. Die Prozeßordnungen der beiden Staaten haben vielmehr noch weitere Bedingungen gesetzt. So müssen zunächst beide Parteien ihr gewohnheitsrechtliches Personalstatut beibehalten haben und die Anwendung ihres Gewohnheitsrechts vor Prozeßbeginn ausdrücklich fordern. Eine option de législation zugunsten des Gewohnheitsrechts bei Mischprozessen oder gar in den Fällen, wo beide Parteien dem statut moderne unterfallen, ist weder in Senegal noch in

42 Penant 1963, S. 548.

43 Cour d'appel, a. a. O

44 Vgl. hierzu Pageard, Penant 1963, S. 486

45 Entsprechendes wird im übrigen auch für die am 3. 2. 1962 verabschiedete Kodifizierung des Familienrechts in der Republik Mali zu gelten haben, vgl. dazu Boubou, Penant 1965, S. 319. 
Niger möglich. In solchen Fällen findet stets Gesetzesrecht Anwendung. Weiterhin ist Gewohnheitsrecht unanwendbar, wenn es gegen den durch das Gesetzesrecht als droit commun gebildeten ordre public verstößt. Zudem hat der Richter in Niger, wie das Gesetz es ausdrückt, „dans le silence et l'obscurité de la coutume" Gesetzesrecht anzuwenden. In Niger wie in Senegal zeigen sich somit Tendenzen, die Anwendbarkeit der Partikularrechte zugunsten des einheitlichen gesetzten Rechts einzuschränken.

Die übrigen Staaten mit einheitlicher Gerichtsbarkeit stehen dem Gewohnheitsrecht aufgeschlossener gegenüber. So kennen z. B. Malit6 und Elfenbeinküste keine Beschränkungen der Anwendbarkeit von Gewohnheitsrecht auf bestimmte Materien - wie dies in Niger und Senegal der Fall ist, ebensowenig Madagaskar, hier allerdings mit den oben skizzierten Ausnahmen. In Gabun, Kongo und Kamerun gelten mangels entgegenstehender Vorschriften die früheren Grundsätze bisher unverändert weiter. Etwas anderes ist auch für Dahomey, Tschad und die Zentralafrikanische Republik nicht ersichtlich ${ }^{47}$.

In allen Staaten besteht die Möglichkeit einer option de législation zugunsten des Gesetzesrechts, die allerdings von beiden Parteien übereinstimmend erklärt werden muß. Diese Regelung entspricht dem Art. 7 des Dekrets vom 3.12.1931, wo dies für das damalige Territorium Französisch-Westafrika ausgesprochen wurde. Das gilt dann jedoch lediglich für den vorliegenden Rechtsstreit. Darüber hinaus ist eine option de statut möglich, wie sie auch schon in der Kolonialzeit bekannt war. In diesem Fall begibt sich der Betreffende ganz oder teilweise seines gewohnheitsrechtlichen Personalstatuts - statut traditionnel - um in der Folge, im Rahmen seines Verzichts, dem Gesetzesrecht zu unterfallen (statut moderne). Dieser Verzicht muß ausdrücklich erklärt werden; für den Betroffenen muß die Möglichkeit einer Wahl tatsächlich bestanden haben. So hat die Cour Suprême in Kamerun entschieden ${ }^{48}$, von einer option de statut zugunsten des Gesetzesrechts könne dann keine Rede sein, wenn zwei kamerunesische Staatsangehörige in Frankreich die Ehe schlössen, da dort eine Ehe nicht anders als in den Formen des Code civil geschlossen werden könne. Dies sei zwar auch in Kamerun selbst möglich, doch könnten die Brautleute dessenungeachtet ihr gewohnheitsrechtliches Personalstatut beibehalten. Somit sei die Ehe selbst - sollte keine nachträgliche option de statut stattfinden - nach ihrem Stammesgewohnheitsrecht zu beurteilen.

e) Bei der in allen Staaten mit einheitlicher Gerichtsbarkeit herrschenden Rechtsmehrheit kommt es notwendigerweise zu Kollisionen zwischen zwei möglicherweise anwendbaren Rechten. Diese Konflikte sind denkbar zwischen Personen des statut moderne und solchen des statut traditionnel wie auch zwischen Personen, die ihrerseits verschiedenen gewohnheitsrechtlichen Personalstatuten unterliegen ${ }^{49}$.

Mehrere Staaten haben positive Rechtsanwendungsnormen zur Lösung derartiger Konflikte gesetzt. In Senegal und Niger werden beide Konfliktsmöglichkeiten gleich behandelt. Hier ergreift somit das gesetzte Recht als droit commun nicht

46 Art. 22, 35 des Gesetzes vom 15. 5. 1961

47 Gesetzliche Regelungen (in der Zentralafrikanischen Republik bis zur Reform 1965 immerhin vorhanden) fehlen nunmehr in allen drei Staaten, obwohl insbes. im Tschad Gewohnheits- und Islamrecht eine außerordentliche Rolle spielen.

48 14. 2. 1961, Penant 1963 , S. 359.

49 Vgl. hierzu allgemein Bouckaert, Les règles de conflit de lois en Afrique noire, Penant 1967 , S. $1 \mathrm{ff}$. 
automatisch Platz, wenn es mit einem Stammesgewohnheitsrecht kollidiert. Vielmehr werden in diesem Fall die Rechte als gleichrangig betrachtet. Einige Lösungen entsprechen denen des Dekrets vom 3. 12.1931, in dem bereits Konfliktsregelungen enthalten waren. Die meisten sind jedoch neu gefaßt. So ist anwendbar bei Ehesachen in Senegal das Recht der Frau, in Niger nur dann, wenn sie nicht Ausländerin ist; dann entscheidet das Recht des Mannes. Bei Testaments- und Erbfolgesachen ist stets das Recht des Erblassers maßgebend. Bei Verträgen ist die Regelung in Niger und Senegal dem Gewohnheitsrecht ohnehin entzogen, so daß nur Gesetzesrecht anwendbar ist.

Bei Schenkungen ist das Recht des Schenkers entscheidend; in sonstigen Fällen, bei denen die Anwendung von Gewohnheitsrecht in Frage kommt, ist das Recht des Beklagten anzuwenden.

Ausführliche Konfliktsregelungen für alle oben erwähnten Konflikte enthalten die Art. 6-11 der madegassischen Ordonnance (No. 60-171) vom 3. 10. 1960. Danach ist für die Ehewirkungen, die Ehescheidung, und die Eltern-Kind-Beziehungen Mannesrecht maßgebend, dagegen richten sich die Ehegültigkeitsvoraussetzungen nach dem Recht jedes der Verlobten. Für Anerkennungen der Vaterbzw. Mutterschaft ist das Recht des vermuteten Elternteils anwendbar, in Adoptionssachen das Recht des Adoptierten, wobei die Fähigkeit zu adoptieren jedoch nach dem Recht des Annehmenden bestimmt wird. In Erbschafts- und Schenkungssachen ist das Recht des Erblassers bzw. Schenkers maßgebend. Schuldrechtliche Beziehungen werden nach dem von den Parteien gewählten Recht beurteilt; fehlt eine dahingehende Bestimmung, so wird wohl - in Übereinstimmung mit der Regelung in den übrigen Staaten - das Recht des Beklagten anzuwenden sein.

Nach der augenblicklichen Rechtslage ${ }^{50}$ greift in Congo bei Mischprozessen litiges mixtes - Gesetzesrecht als droit commun ein. Bei Konflikten zwischen zwei Gewohnheitsrechten sind ebenfalls ausdrückliche Regelungen, teilweise in Anlehnung an das oben zitierte Dekret, entstanden. Bei Ehesachen entscheidet das Recht, nach dem die Ehe zustande gekommen ist, hilfsweise das Recht des Mannes; im Hinblick auf die Kinder allerdings das für sie günstigste Recht. Bei Testaments- und Erbfolgesachen ist das Recht des Erblassers anzuwenden, wobei die Bestimmungen des Erblassers über das auf die Erbfolge anwendbare Recht zu beachten sind; bei Schenkungen ist das Recht des Schenkers maßgebend, bei Verträgen die lex loci contractus. Für andere, ebenfalls dem Gewohnheitsrecht unterfallende Materien besteht keine Regelung.

Die übrigen Staaten mit einheitlicher Gerichtsbarkeit haben diese Fragen nicht positiv geregelt. Auch hier wird man eine Weitergeltung der während der Kolonialzeit erlassenen Bestimmungen annehmen können, insbesondere wird bei Mischprozessen auch hier Gesetzesrecht als droit commun eingreifen. Soweit auffindbar, hat sich die Rechtsprechung in diesen Staaten dazu noch nicht gegenteilig geäußert.

Zusammenfassend läßt sich feststellen, daß bis auf Guinea in den Staaten mit ein. heitlicher Gerichtsbarkeit die Zweiteilung der Statuten fortwirkt. Es wird versucht, dieser Rechtsmehrheit mit einheitlichen Kodifizierungen $\mathrm{zu}$ begegnen, dieses Ziel kann jedoch erst in noch nicht absehbarer Zeit erreicht werden.

$50 \mathrm{Ob}$ die politische Entwicklung in Congo (Brazzav.), das am 3. 1. 1970 die sechste Verfassung seit der Unabhängigkeit beschlossen hat und sich nunmehr zum Marxismus-Leninismus bekennt, eine ähnliche Rechtsentwicklung wie in Guinea nach sich ziehen wird, bleibt abzuwarten. 
Weiterhin zeichnen sich innerhalb der Staaten des „système unitaire“ zwei Tendenzen im Hinblick auf die Stellung des Stammesgewohnheitsrechts ab. So ist beispielsweise in Senegal, Niger, Kamerun und Gabun - die Tendenz erkennbar, den Primat des Gesetzesrechts durchzusetzen, was sich allgemein darin äußert, daß diese Staaten den gewohnheitsrechtskundigen Beisitzern die Richterqualität abgesprochen haben, diese somit nur beratende Funktionen ausüben. Im besonderen haben von diesen Staaten Niger und Senegal die Anwendbarkeit von Gewohnheitsrecht auf wenige, genau bezeichnete Fälle beschränkt. Hier kann von einer "justice légale" gesprochen werden. Eine "justice populaire"51 wird dagegen zumindest in den drei Staaten Mali, Elfenbeinküste und Congo (Brazzaville) ausgeübt. Hier kommt den Beisitzern beschließende, richterähnliche Funktion zu; eine Beschränkung der Anwendbarkeit von Gewohnheitsrecht wie in Niger und Senegal findet nicht statt.

51 Vgl. Pageard, Penant 1963, S. 489. 